\title{
SUPERNOVA REMNANTS IN GIANT HII REGIONS
}

You-Hua Chu

Astronomy Department, University of Illinois

1011 W. Springfield Avenue, Urbana, IL 61801

Robert C. Kennicutt, Jr.

Astronomy Department, University of Minnesota

116 Church Street, S.E., Minneapolis, MN 55455

\section{Introduction}

Giant HII regions contain large numbers of massive stars, and hence are expected to contain large numbers of SNRs. Until recently, however, only a few SNRs have been identified in extragalactic giant HII regions. Moreover, most of these SNRs are located at the outskirts of HII regions, instead of the core where most of the stars are located. The low detection rate and the outlying locations of the SNRs may be due to: 1) observational difficulties - the background HII regions are much more luminous than the SNRs in both optical line emission and radio continuum; 2) intrinsic invisibility of SNRs - stellar wind and SNRs may have created a supershell (Mac Low and McCray 1987), and the core of a giant HII region is filled with hot tenuous coronal gas; or 3 ) a genuine deficiency of supernovae and SNRs in the HII regions (Sramek and Weedman 1986).

We have begun to use high dispersion emission-line spectroscopy to supplement the traditional methods in the search for SNRs in large HII regions. Such observations offer a very sensitive means of detecting SNRs in these regions, and the potential of studying the interaction of remnants with the interstellar medium in complex environments. Here we present a brief progress report on this program, and summarize what is known about SNRs in the largest HII regions in several nearby galaxies.

\section{SNRs in Giant HII Regions}

SNRs are actually present in the largest HII regions of many nearby spiral and irregular galaxies. Table 1 lists nearby galaxies with confirmed SNRs in giant HII regions. These include previously identified SNRs, and objects identified in our spectroscopic observations (SMC, M101).

The SNR W49B in W49, probably the largest HII region in the Galaxy, is so heavily obscured that no optical observations are possible. The radio properties of $\mathrm{W} 49 \mathrm{~B}$ are typical for SNRs: a nonthermal shell source with a spectral index of -0.5 (Green, Baker, and Landecker 1975). 
Table 1. SNRs in Giant HII Regions

\begin{tabular}{|c|c|c|c|c|}
\hline Galaxy & $\begin{array}{l}\text { Distance } \\
(\mathrm{kpc})\end{array}$ & HII Region & $\begin{array}{l}\text { Ionizing Flux } \\
\text { (ion. photon/s) }\end{array}$ & SNR \\
\hline$\overline{\text { Galaxy }}$ & - & W49 & $7 \times 10^{50}$ & W49B \\
\hline LMC & 55 & 30 Dor & $\times 10^{51}$ & 30 Dor $B$ \\
\hline SMC & 70 & N66 & $\times 10^{50}$ & anon \\
\hline M33 & 720 & NGC 604 & $1.5 \times 10^{51}$ & M33-16 \\
\hline M101 & 6000 & NGC 5471 & $1.7 \times 10^{52}$ & (3 SNRs) \\
\hline
\end{tabular}

30 Dor B is the only positively identified SNR in 30 Doradus (the largest HII region in the Large Magellanic Cloud), although there is kinematic evidence for several other possible SNRs, as discussed later. 30 Dor $B$ is located at $-100 \mathrm{pc}$ from the core of the HII region. Its velocity structure is well described by a complete but irregular she11, expanding at $-180 \mathrm{~km} / \mathrm{s}$. To the east of this shell, there is high-velocity material moving at $+120 \mathrm{~km} / \mathrm{s}$ relative to the average background nebular velocity; the relation between this high-velocity material and the SNR 30 Dor B is not clear. 30 Dor B is Crab-like in the radio, but its slower expansion velocity $(-180 \mathrm{~km} / \mathrm{s})$ and larger linear size (diameter -10 pc) make it much older than the Crab. We derive a dynamic age of about $10^{4} \mathrm{yr}$, which is considerably longer than the previous estimates (Long and Helfand 1979; Gilmozzi et al. 1983). The radio emission is more extended to the west of the optical components (the [S II]/H $\alpha$ enhanced region), and there is high velocity material to the east of the remnant. The statistics of Crab-like SNRs are minimal enough to prohibit meaningful comparisons between 30 Dor $B$ and isolated SNRs of similar type.

N66 is the largest HII region in the Small Magellanic Cloud. It contains an X-ray source and nonthermal radio sources (Ye and Turtle, 1986, private communication), but no optical SNRs were previously identified. The comparison of a radio map at $843 \mathrm{MHz}$ (Mills et al. 1982 ) and a short-exposure $\mathrm{H} \alpha$ picture of the core region in $\mathrm{N} 66$ shows that the brightest peaks in the radio map do not have corresponding $\mathrm{H} \alpha$ features. A long-slit $\mathrm{H} \alpha$ echellogram taken at this region shows highvelocity clumps, up to $-+170 \mathrm{~km} / \mathrm{s}$ with respect to the background HII region velocity. The coincidence of nonthermal radio emission and an $\mathrm{X}$-ray source at this fast expanding shell makes it a good SNR candidate.

NGC 604, the brightest HII region in M33, contains a known SNR (D'Odorico, Dopita, and Benvenuti 1980). SNRs in M33, at a distance of $720 \mathrm{kpc}$, are hardly resolved by ground based telescopes. The echellogram of NGC 604 shows expanding shells with a range of sizes and velocities in the central HII region, and an unresolved broad component in the SNR. The SNR has similar surface brightness and kinematic structures as the other SNRs in M33 (Blair, Chu, and

Kennicutt 1987). Its radio flux density is about $0.6 \mathrm{mJy}$ at $1420 \mathrm{MHz}$ (Sramek and Weedman 1986), within the typical range for the other SNRs in M33 (D'Odorico, Goss, and Dopita 1982). 
NGC 5471, the brightest HII region complex in M101, contains at least 3 SNR candidates (Chu and Kennicutt 1986). All have large velocity widths $(300-460 \mathrm{~km} / \mathrm{s}$ at the base) which are similar to those of isolated SNRs in M33 and the LMC. In addition, spectrophotometry and CCD images show enhanced [SII] emission at the SNRs. The most remarkable properties of these SNRs are their high optical and radio luminosities. The brightest SNR in NGC 5471 B, first identified by Skillman (1985), has an Ha luminosity of $6.3 \pm 3 \times 10^{38} \mathrm{erg} / \mathrm{s}$, and radio flux density of $2.3 \pm 0.2 \mathrm{mJy}$ at $1415 \mathrm{MHz}$. The size (<30 pc), kinematic structures, and Ho luminosity imply a middle-aged SNR ( - a few $x 10^{4} \mathrm{yr}$ ), but the radio luminosity is only matched by very young SNRs (a few $x 10^{2} \mathrm{yr}$ ). The SNR in NGC 5471 A probably has similar properties. It is possible that these properties resulted from the peculiar interstellar environment in the dense core of NGC 5471; it is also possible that multiple SNR events are seen there. However, it must be noted that any search for SNRs at this large a distance will detect only unusually bright objects. Similar SNRs in other giant HII regions have to be found and studied before this issue can be settled.

\section{How to Search for SNRs in Giant HII Regions}

Conventionally, SNRs are identified using [SII]/Ho imagery, $\mathrm{X}$-ray, and radio surveys. However, in distant luminous HII regions the environments are so unusual that unconventional methods have to be used to search for SNRs. [S II]/Ho imagery may be effective if the background HII region is not too luminous, or if the SNRs are very luminous, especially if $C C D$ detectors are used. The radio surveys are good for picking out nonthermal sources if the spatial resolution is sufficient. As we have demonstrated in N66 and NGC 5471, high dispersion 2-D spectroscopy is an alternative and a very effective way to search for SNRs in complex emission-line regions. The combination of these methods, especially a high resolution radio survey with high dispersion 2-D spectroscopy, is probably the most effective way to search for SNRs in giant HII regions.

Why bother searching for SNRs in such complex, high background regions when there are plenty of relatively isolated, easily observed SNRs available? For the study of the interaction of SNRs with the interstellar medium, the remnants in giant HII regions provide an opportunity to investigate how the evolution of SNRs are altered in the relatively dense ionized nebular environment. Conversely it should be possible to establish whether the SNRs significantly influence the global kinematics and dynamics of the giant HII regions and their surroundings. Very luminous and energetic SNRs, such as the object in NGC $5471 \mathrm{~B}$, may require unusually massive progenitor stars, and it is of great interest to identify and measure a larger sample of these unusual objects. Finally the detection and study of SNRs in regions such as giant HII regions, starburst galaxies, and galactic nuclei is important in its own right, since supernovae may be dominant contributions to the energetics and radio emission of these objects. 
In order to address some of these questions we are following up our initial survey on several fronts. Comparable high-dispersion spectra of isolated SNRs in the Magellanic Clouds and M33 are being obtained (Chu and Kennicutt 1987; Blair, Chu, and Kennicutt 1987), in order to compare the kinematic properties of the SNRs in HII regions with isolated ones. These data also provide the best available data on the integrated kinematic properties and nebular environments of SNRs in general. We also hope to enlarge our sample of giant HII regions surveyed, in order to set firmer limits on the number of very luminous SNRs. Finally we have obtained high-resolution data at multiple positions in the nearest supergiant HII region, 30 Doradus in the LMC, in order to conduct a sensitive search for SNRs (and other kinematic structures) in the dense nebular core of this HII region.

\section{Fast Expanding Shells in the Core of 30 Doradus}

Our long-slit echelle spectra at several positions in 30 Doradus reveal a number of faint shells, expanding at $150-200 \mathrm{~km} / \mathrm{s}$, in the bright nebular core. Features with similar expansion velocities have been reported and interpreted as wind-blown bubbles by Meaburn (1984). We find these shells very intriguing, since expansion velocities of isolated wind-blown bubbles have never been observed to be greater than $100 \mathrm{~km} / \mathrm{s}$. These expansion velocities are more in accord with the other SNRs in the Magellanic Clouds (Chu and Kennicutt 1987), and the presence of dense high-velocity clumps in the shells is also much more characteristic of SNRs. From kinematic evidence alone, these fast expanding shells could conceivably be either stellar wind blown bubbles or SNRs. It is important to examine closely the stellar content and the radio properties of these shells in order to determine their real nature.

\section{References :}

Blair, W. P., Chu, Y.-H., and Kennicutt, R. C. 1987, in this volume. Chu, Y.-H., and Kennicutt, R. C., 1986, Ap. J., 311, 85.

Chu, Y.-H., and Kennicutt, R. C., 1987, in preparation.

D'Odorico, S., Dopita, M. A., and Benvenuti, P. 1980, Astr. Ap., $40,67$.

D'Odorico, S., Goss, W. M., and Dopita, M. A., 1982, M. N. R. A. S., 198,1059 .

Gilmozzi, R., Murdin, P., Clark, D. H., and Malin, D. 1987, M. N. R. A. S., $202,927$.

Green, A. J., Baker, J. R., Landecker, T.L., 1975, Astr. Ap., 44, 187. Long, K. S., and Helfand, D. J. 1979, Ap. J. (Letters), 234, L77. Mac Low, M.-M., and McCray, R. 1987, Ap. J., in press.

Meaburn, J. 1984, M. N. R. A. S., 211, 521.

Mills, B. Y., Little, A. G., Durdin, J. M., and Kesteven, M. J., 1982, M. N. R. A. S., 200, 1007.

Skillman, E. D. 1985, Ap. J., 290, 449.

Sramek, R. A., and Weedman, D. W., 1986, Ap. J., 302, 640. 